\title{
LOS SISTEMAS DE APOYO EN LA TOMA DE DECISIONES
}

\author{
SUPPORT SYSTEMS IN DECISION MAKING
}

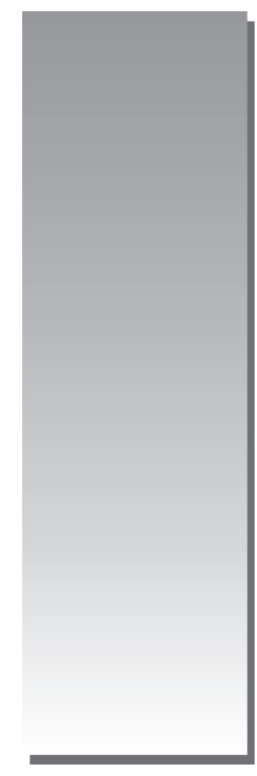

Félix Armando Rivera León*

felix_arl@hotmail.com

\begin{abstract}
RESUMEN
Los sistemas de apoyo en la toma de decisiones (SSD) son sistemas informáticos interactivos que ayudan a los encargados de tomar decisiones utilizando datos y modelos para resolver problemas no estructurados" (Spraguey Carlson).

El uso de herramientas tradicionales basadas en el desarrollo de aplicaciones sobre sistemas de gestión de bases de datos para obtener información estratégica para la toma de decisiones presenta inconvenientes de diferente índole, como tiempos de respuestas extremadamente lentas, dificultad o imposibilidad de expresar una consulta compleja y dificultad para la obtención de resultados que recopilen información de fuentes diversas.
\end{abstract}

Palabras claves: Sistemas de apoyo, toma de decisiones.

\begin{abstract}
Support Systems in Decision Making (SSD) "are interactive computer systems that help decision makers use data and models to solve unstructured problems" (Spraguey Carlson).

The use of traditional tools based on application development management systems databases for strategic information for decision drawback presents of various kinds, such as extremely slow response time, difficulty or inability to express a complex query, and difficulty obtaining results that collect information from various sources
\end{abstract}

Keywords: Support systems; decision-making.

\footnotetext{
* Dr. en Ciencias Contables y Empresariales- UNMSM, magíster en Administración con mención en Gestión Empresarial, contador público colegiado. Profesor principal de la Facultad de Ciencias Administrativas - UNMSM. Autor de libros y revistas sobre temas de su especialidad, auditoría. Ha desempeñado diversos cargos administrativos en la facultad y en diferentes entidades del sector público y privado.

1. R. H. and E.D. Carlson Building Effetive Decision Support System. Englewood Cliffs, NJ, Prentice-Hall Inc 1982, appeared in DSS News, february 11, 2001, Vol 2, No. 4
} 


\section{INTRODUCCIÓN}

Parece que nos encontramos al borde de otra "era" en el inexorable avance de los sistemas de información computarizada para organizaciones. Designados con el término: sistemas de apoyo en la toma de decisiones (SSD), estos sistemas están recibiendo reacciones que los califican desde un "adelanto importante" hasta de "simplemente otro término más para llamar la atención.

Un punto de vista es que la evolución natural de la tecnología informativa y su uso dentro del contexto organizacional ha avanzado desde el PED (procesamiento electrónico de datos) y SIG (sistemas de información gerencial), hasta la actual acometida de los SSD (sistemas de soporte a la decisión). De acuerdo a esta opinión, los SSD parten desde donde terminan los SIG.

Una visión contraria califica a los SSD como un importante subconjunto de lo que han sido y seguirán siendo los SIG. Y otro punto de vista reconoce un tipo de sistema que se ha estado desarrollando durante varios años y que "ahora ya tiene nombre". Mientras tanto, los escépticos sospechan que el término SSD es totalmente otra "palabrita atractiva" para justificar la siguiente ronda de visitas de los vendedores.

\section{DEFINICIÓN, EJEMPLOS Y CARACTERÍSTICAS}

Los conceptos relacionados con el SSD fueron articulados por vez primera a principios de la década del setenta por Michael S. Scott Morton, bajo el término "sistemas de decisión gerencial". Unas cuantas firmas y estudiosos empezaron a desarrollar e investigar los SSD, que se caracterizaron como sistemas interactivos basados en computadoras que ayudan a quienes toman decisiones a utilizar datos y modelos para resolver problemas no estructurados. La contribución especial de los SSD provino de estas palabras claves. Dicha definición resultó lo suficientemente restrictiva como para que solo unos cuantos sistemas reales pudieran satisfacerla completamente. Algunos autores recientemente han ampliado la definición de los SSD para incluir a cualquier sistema que contribuye a la toma de decisiones; de esta manera, el término puede aplicarse a todos los procesos, excepto, el proceso transaccional. Un grave problema en cuanto a las definiciones es que las palabras tienen una determinada "validez intuitiva": cualquier sistema que apoye una decisión, de la manera que sea, es un "sistema de soporte a la toma de decisiones", simplemente un "sistema de soporte de decisiones" (SSD).

\section{SSD vs. SIG}

Gran parte de la dificultad y controversia con los términos como SSD y SIG puede encontrarse en las diferencias de una definición académica o teórica, o en una definición "connotativa". La primera es cuidadosamente articulada por gente que escribe libros y publica artículos en revistas especializadas. La segunda evoluciona de los verdaderos desarrollos y sus prácticas y es fuertemente influenciada por las experiencias personales que ha tenido el usuario del término con el tema. Es esta la definición connotativa de $\mathrm{PED} / \mathrm{SIG} / \mathrm{SSD}$ que se emplea para justificar la aseveración de que un SSD es un avance evolutivo que va más allá del SIG.

Esta visión puede ser expresada utilizando la figura 1, un organigrama sencillo, como modelo de una organización. El PED fue aplicado por primera vez a los niveles operacionales más inferiores de la organización para automatizar el papeleo. Sus características son:

- Concentración en los datos, almacenamiento, procesamiento y flujos a nivel operacional.

- Un eficiente procesamiento transaccional.

- Corridas de computadoras; programadas y optimizadas.

- Archivos integrados para trabajos conexos.

- Informes resumidos para la gerencia.

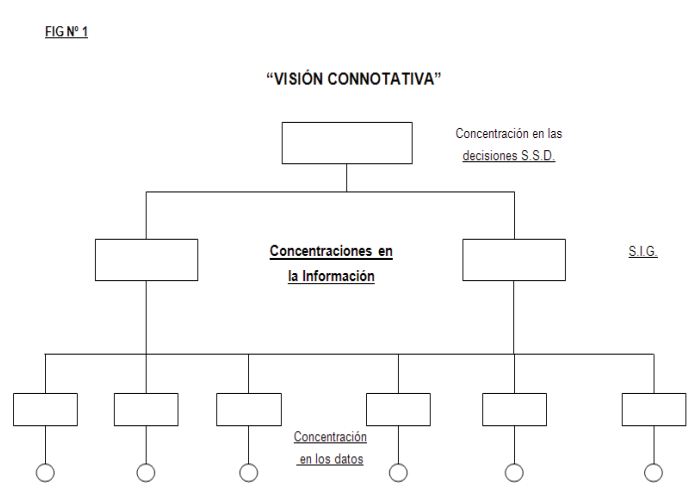

En los años recientes, el nivel de actividad del PED en muchas firmas se ha convertido en una facilidad productiva bien aceitada y eficiente para el procesamiento de transacciones. 
El enfoque de los SIG elevó el foco de las actividades de los sistemas de información, poniendo mayor énfasis en la integración y planificación de la función de los sistemas de información. En la práctica, las características de los SIG incluyen:

- Concentración en la información, dirigida a los gerentes intermedios.

- Flujo estructurado de la información.

- Integración de los empleos de PED, de acuerdo a su función comercial, tales como SIG de producción, SIG de marketing, SIG de personal, etc.

- Generación de preguntas y reportes, usualmente base de datos.

La era de los SIG aportó un nuevo nivel de información que servía a las necesidades de la gerencia, pero seguía estando muy orientada a los flujos de información y archivos de datos, además de basarse en ellos.

De acuerdo con la visión connotativa. un SSD se concentra en un nivel más alto de la organización, no enfatizando las siguientes características:

- Concentración en las decisiones, dirigido a los gerentes y ejecutivos de alto nivel que toman decisiones.

- Énfasis en la flexibilidad, adaptabilidad y respuestas rápidas.

- Iniciación y control del usuario.

- Soporte para los estilos personales que cada gerente en particular tiene para tomar decisiones.

Esta visión connotativa y evolutiva tiene cierto crédito, porque corresponde aproximadamente a los desarrollos que se han visto en la práctica a través del tiempo. Un reciente estudio determinó que los gerentes de SIG podían distinguir el nivel de avance de sus sistemas de aplicación utilizando criterios similares a los mencionados líneas arriba. Muchas instalaciones con aplicaciones de tipo SIG, planificaron desarrollar aplicaciones con características de tipo SSG. Sin embargo, la visión "connotativa" tiene serias deficiencias y definitivamente es engañosa a la hora de desarrollar más un SSD.

- Implica que el apoyo para la toma de decisiones solo se necesita en los niveles más altos. En realidad, dicho apoyo se requiere en todos los niveles gerenciales de la organización.
- La toma de decisiones que ocurre en los diversos niveles con frecuencia debe ser coordinado. Por lo tanto, una dimensión importante del apoyo para la toma de decisiones es la comunicación y coordinación entre aquellos que toman decisiones a lo largo de los diferentes niveles de la organización, así como también al mismo nivel.

- Implica que el apoyo para la toma de decisiones es lo único que necesitan los gerentes generales del sistema de información. En realidad, la toma de decisiones es tan solo una de las actividades gerenciales que se benefician con el apoyo para los sistemas de información.

También existe el problema de que muchos profesionales en sistemas de información, especialmente en SIG, no están dispuestos a aceptar la obtusa visión connotativa del término SIG. Para nosotros, el SIG se refiere a todo el conjunto de actividades y sistemas que se requieren para manejar, procesar y utilizar información como un recurso de la organización.

\section{LA VISIÓN TEÓRICA}

Para poder considerar el rol apropiado de un SSD dentro del contexto general de los sistemas de información, a continuación mencionamos las características de los fines y objetivos más amplios de la función de los sistemas de información organizacional:

Dedicado a perfeccionar el rendimiento de los trabajadores de la organización a través de la aplicación de tecnología de la información (TI).

El objetivo final de los sistemas de información es mejorar el rendimiento, no el almacenamiento de datos, la producción de informes ni siquiera "el suministro de la información correcta a la persona correcta en el momento correcto". El objetivo final debe ser observado en cuanto a la habilidad que tienen los sistemas de información para apoyar el rendimiento mejorado de la gente de las organizaciones.

- Los trabajadores capacitados o cultos son la clientela. Este grupo incluye a gerentes profesionales, analistas de staffy oficinistas cuya principal responsabilidad laboral es manejar la información de alguna manera.

- Las organizaciones son el contexto. El enfoque está en manejar la información en todo tipo de organizaciones que persiguen metas. 
- La aplicación de la tecnología informativa es el desafío y la oportunidad que tienen los profesionales en sistemas de información para los propósitos y dentro de los contextos enunciados anteriormente.

A fines de la década del setenta, Robert Head utilizó un triángulo como modelo visual para caracterizar al SIG en sentido global y amplio. Se ha convertido en una manera clásica de ver las dimensiones de un sistema de información. La dimensión vertical representaba los niveles de gerencia y la dimensión horizontal representaba las principales áreas funcionales de la organización comercial. Posteriormente, otros autores agregaron el procesamiento transaccional como una base sobre la cual descansaba todo el sistema. E1 resultado fue un modelo bidimensional de un SIG, en el amplio sentido del término: todas las actividades comprendidas por el sistema de información de una organización. En la figura 2 apreciamos una extensión adicional del triángulo básico que nos ayuda a describir el concepto de rol potencial de un SSD. La dimensión de profundidad muestra los principales "subsistemas" tecnológicos que sirven de apoyo para las actividades de los trabajadores profesionales.

Aquí se muestran tres aspectos importantes, pero podrían haber más. El sistema estructurado de reportes incluye los requeridos para administrar y controlar la organización y satisfacer las necesidades informativas de los sectores externos. Ha ido evolucionando desde los esfuerzos en PED y SIG y en el sentido estricto del término, durante muchos años. Los sistemas para apoyar las necesidades de comunicación que tienen las organizaciones están evolucionando rápidamente debido a los avances en telecomunicación, con un fuerte ímpetu proveniente de la automatización de las oficinas y el procesamiento de palabras. El SSD parece estar evolucionando de la unión de la tecnología informativa con los enfoques científicos de investigación/administración de operaciones, a manera de modelación interactiva.

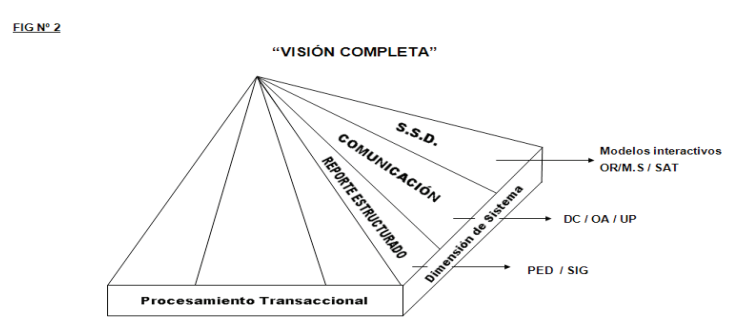

\section{TRES NIVELES DE TECNOLOGÍA}

Es conveniente identificar tres niveles de hardware/software (equipo físico/programas y rutinas) que han sido incluidos bajo el nombre de SSD. Son empleados por personas con diferentes niveles de capacidad técnica y varían en naturaleza y ámbito de tareas en las que pueden ser aplicados.

\section{SSD específico}

El sistema que en realidad logra el trabajo podría denominarse el SSD específico. Es una "aplicación" de los sistemas de información, pero con características que la hacen muy diferente de una típica aplicación de procesamiento de datos. Es el hardware/software a que permite a una o varias personas específicas que toman decisiones manejar un conjunto determinado de problemas conexos. Un ejemplo inicial es el sistema de administración de cartera, que también está descrito en el primer libro importante sobre SSD, escrito por Keen y Scott Norton. Otro ejemplo sería el sistema de asignación de rondas policiales, utilizado experimentalmente por la Ciudad de San José, California. Este sistema permitía que un oficial de policía trazara un plano y pidiera datos por área geográfica, mostrando los llamados de ayuda policial, niveles de actividad, tiempo de servicio, etc. La capacidad gráfica interactiva del sistemas permitía al oficial manipular los mapas, zonas y datos para ensayar rápida y sencillamente una variedad alternativa para las rondas policiales. En efecto, el sistema proporcionaba las herramientas para ampliar el criterio de un gerente. A propósito, un experimento posterior trató de aplicar un modelo tradicional de programación lineal al problema. La solución fue menos satisfactoria que la proporcionada por el oficial de policía.

\section{Generador de SSD}

El segundo nivel de tecnología podría denominarse generador de SSD. Este es un "paquete" de hardware y software relacionados que permiten una serie de capacidades para construir, rápida y sencillamente, un SSD específico. Por ejemplo, el sistema de rondas policiales descrito líneas arriba fue construido según el Sistema de Exhibición y Análisis de Geodatos (GADS) , un sistema experimental desarrollado en los Laboratorios de Investigación de la IBM de San José. Mediante la carga de diferentes mapas, datos, selecciones a la carta, y procedimientos y líneas de mando, los GADS fueron posteriormente utilizados para cons- 
truir un SSD específico para apoyar la localización de los técnicos que reparaban copiadoras IBM. El desarrollo de esta nueva "aplicación" tomó poco menos de un mes.

Otro ejemplo de un generador de SSD es el sistema de información ejecutiva (EIS), comercializado por los servicios de computo de la Boeing. EIS es un conjunto integrado de capacidades que incluye la preparación de reportes, capacidad de averiguación, lenguaje de modelación, mando para la exhibición gráfica, así como un conjunto de subrutinas de análisis estadístico y financiero. Todas estas capacidades han existido individualmente durante algún tiempo.

La contribución especial del EIS es que estas capacidades existen ahora a través de un lenguaje de mando común que actúa a base de un conjunto de datos comunes. El resultado es que los EIS pueden ser utilizados como generadores de SSD, especialmente para que un SSD específico que ayude en casos en donde se tenga que tomar decisiones financieras.

El crecimiento evolutivo hacia los generadores de SSD ha provenido de lenguajes de propósitos especiales. En realidad, la mayoría de los sistemas de software que podrían emplearse como generadores están evolucionando de lenguajes ampliados de planificación o lenguajes de modelación o tal vez con el agregado de que pueden preparar reportes y exhibir gráficos. El Sistema Interactivo de Planeamiento Financiero (IFPS), comercializado por Execucom Systems de Austin, Texas, y EXPRESS, lanzado por TYMSHARE, constituyen buenos ejemplos.

\section{Herramientas del SSD}

El tercer nivel y el más fundamental de la tecnología aplicada al desarrollo de un SSD podría denominarse Herramientas del SSD. Estos son elementos de hardware o software que facilitan el desarrollo de un SSA específico o de un generador de SSD. Esta categoría de tecnología ha tenido grandes avances recientemente, incluso la creación de nuevos lenguajes para fines especiales, mejoras en sistemas operativos que apoyen los enfoques conversacionales, equipo con gráficas a color, software de apoyo, etc. Por ejemplo, el sistema GADS, descrito anteriormente, fue escrito en FORTRAN utilizando un paquete experimental de subrutinas gráficas como el primer software para el manejo de diálogos, un monitor a color para el examen de fondo o trama realizado para uso de laboratorio y un poderoso sistema interactivo de extracción de datos de administración de base de datos.

\section{Evolución de roles en SSD}

Probablemente, los tres niveles de tecnología van a ser utilizados a través del tiempo en el desarrollo y operación de un SSD. Sin embargo, se están presentando algunos avances interesantes en los roles que juegan los gerentes y técnicos.

En el contexto anterior se presentan con cinco roles dispersos a lo largo de los tres niveles:

- El gerente o usuario es la persona que afronta el problema o la decisión, la que debe tomar una acción y ser responsable de las consecuencias.

- El intermediario es la persona que ayuda al usuario, tal vez simplemente como asistente de oficina, a presionar los botones del terminal, o tal vez como un "asistente administrativo", más importante, el terminal, o tal vez como un asistente valioso, que actúa en reciprocidad y formula sugerencias.

- El constructor o facilitador de los SSD reúne las capacidades necesarias del generador de los SSD para "configurar" él SSD específico con el cual el intermediario/ usuario interactúa en forma directa. Esta persona debe estar familiarizada de algún modo con el área de problema y sentirse en comodidad con los componentes y capacidades de la tecnología del sistema de información.

- El apoyador técnico desarrolla capacidades adicionales del sistema de información cuando se necesitan como parte del generador. Nuevas bases de datos, nuevos modelos de análisis y formatos para el despliegue de datos adicionales serán desarrollados por la persona que desempeñe este cargo. Esto exige una mayor familiaridad con la tecnología y un menor conocimiento del área del problema o de aplicación.

- El forjador de herramientas desarrolla nuevas tecnologías, nuevos lenguajes, nuevo hardware, así como software, mejora la eficiencia de enlaces articulados entre subsistemas, etc. 


\section{Diseño interactivo}

El resultado es que los cuatro pasos más importantes en el típico proceso de desarrollo de sistemas - análisis, diseño, construcción, implementación se combinan en un paso único el cual es repetido constantemente. Varios nombres se desenvuelven para describir este proceso, entre ellos el montaje experimental, el enfoque evolutivo y salida intermedia.

La esencia del enfoque es que el gerente y el constructor concuerdan en un subproblema pequeño pero significativo, y luego diseñan y desarrollan un sistema inicial para apoyar la toma de decisión que él exige. Tras un corto período de uso, por ejemplo unas cuantas semanas, el sistema es evaluado, modificado y expandido incrementalmente. Este ciclo se repite de tres a seis veces en el curso de unos pocos meses, hasta que alcanza un sistema relativamente estable, el cual apoya la toma de decisiones para un grupo de tareas. La palabra "relativamente" es importante debido a que, si bien la frecuencia «su extensión de cambio disminuirá, nunca será estable. El sistema siempre estará cambiando, no como un mal necesario en respuesta a cambios ambientales impuestos, sino como una estrategia consciente de parte del usuario y constructor.

Finalmente, un SSD debería ser fácil de usar. Una variedad de términos ha sido usada para describir características, incluyendo flexibilidad, usuario amistoso, nada de amenazas, etc. La importancia de esta característica es subrayada por la amplitud discrecional de la clientela del SSD Aunque algunos sistemas que requieren pesado apoyo organizativo o apoyo de grupo puedan limitar en algo la discreción, el usuario de un SSD tiene mucha más amplitud para hacer caso omiso al pasar alrededor del sistema, que el usuario de un sistema de desempeño más tradicional o sistemas de informe requerido. Por tanto, un SSD debe "ganar" la lealtad de sus usuarios por su condición de conveniente y valioso.

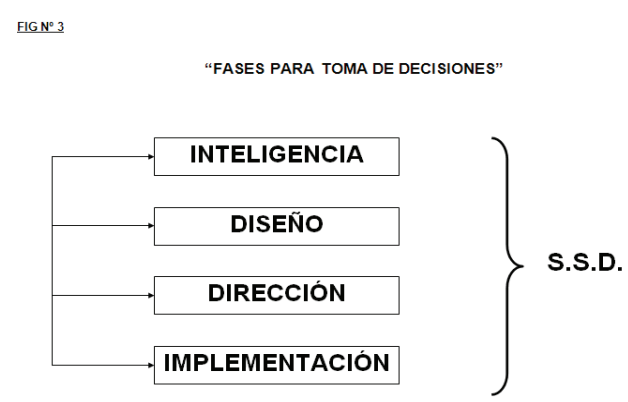

\section{Manejo de Datos}

La mayoría del trabajo significativo en el área de administración de base de datos, durante los años recientes, tiende hacia al procesamiento de transacciones, contra grandes bases de datos. Las grandes DBMS generalmente poseen capacidades de pregunta/respuesta y de preparación de reporte flexible, pero su mayor contribución ha sido en la reducción de los costos de mantenimiento de programas, mediante la separación de programas de aplicación y las definiciones de datos. En cambio, el trabajo sobre los DBMS ha tenido por lo general una concepción algo cándida del usuario y de sus exigencias. El usuario de un SSD no se sentirá satisfecho solamente con la capacidad de emitir un conjunto de cometidos de recuperación que selecciona ítems de la base de datos o que estos aparezcan en una pantalla con posibilidades, definir formatos y encabezamientos. El usuario de un SSD necesita interactuar repetida y creativamente con un conjunto relativamente pequeño de datos. El usuario puede necesitar solamente de 40 a 100 variables de datos, pero deben ser los correctos; y lo que es correcto puede cambiar de un día al otro, o de una semana a la siguiente. Entre los datos requeridos, probablemente se incluirán datos de series cronológicas, que no son manejados en forma amplia por los SBMS típicos. Se necesita mejores modos de manejar b y coordinar series cronológicas, así como mecanismos para capturar, procesar y rotular datos de probabilidades, o de carácter valorativos. Se requieren también mejores formas de extracción de datos de los archivos existentes y capturar datos de fuentes no computarizados previamente. El área crítica de la extracción de datos no computarizados previamente. El área crítica de la extracción de datos con respuesta rápida, que permita adiciones y supresiones a la base de datos del SSD, provenientes de la base de datos de grandes transacciones, fue una contribución mayor del trabajo del GADS

En suma, el significativo desarrollo en la tecnología de base de datos necesita enfocarse y ampliarse a algunas áreas clave, para servir directamente a las necesidades de un SSD.

\section{Administración de modelos}

El área de creación y manejo de modelos puede tener la más grande contribución potencial para el SSD. Hasta ahora la capacidad analítica proporcionada por sistemas ha evolucionado desde 
subrutinas estadísticas o de análisis financiero, que se pueden convocar desde un lenguaje común de mando. Más recientemente, los lenguajes modeladores facilitan un modo de formular interrelaciones entre variables, de un modo que permite la creación de simulación de modelos del tipo "que pasa si"... Como ya hemos anotado antes, muchos de los actuales generadores SSD viables han evolucionado a partir de estos esfuerzos. Las formas iniciales de "manejo de modelos" parecen estar evolucionando con el mejoramiento de algunos lenguajes modeladores, lo que permite que un modelo de este tipo sea utilizado para análisis de sensibilidad o búsqueda de metas, mediante la especificación de variables, objetivo y de flexibilidad.

El área de administración de modelos posee también el potencial para traer algunas de las contribuciones de inteligencia artificial (IA) a lograrse con un SSD. EMYCIN, un sistema para apoyar los diagnósticos médicos, se basa en "reglas de producción”, en el sentido de inteligencia artificial, que son las que desempeñan los roles de modelo a realizar funciones de analíticas y de guía de la decisión. Una caracterización más general de "manejo del conocimiento" como un medio de manejar modelos y datos ha sido también explorada tentativamente. Un trabajo más reciente propone el uso de una versión de sistemas semánticos para la presentación del modelo. Aunque este último trabajo es promisorio, la investigación en inteligencia artificial ha mostrado que el enfoque del sistema semántico es relativamente ineficiente con la tecnología de hoy. Lo más probable es que las capacidades utilizables en el manejo del modelo, evoluciones a partir de los lenguajes modeladores, de los expandidos enfoques de las subrutinas, y en algunos casos, de las reglas de producción de inteligencia artificial, lo sobrepasan.
COMPONENTES DE UN S.S.D.

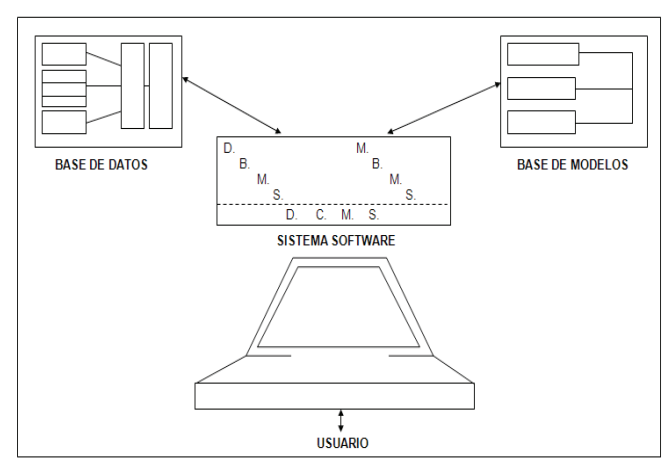

\section{CONCLUSIÓN}

1. Los SSD ayudan a los gerentes a tomar decisiones para tratar de resolver problemas semiestructurados; asímismo, apoyan al juicio del gerente en lugar de reemplazarlo, mejorando su eficiencia en la toma de decisiones.

2. Se podría decir que un SSD es un sistema de información que combina datos y modelos analíticos, sofisticados o herramientas de análisis de datos para apoyar la toma decisiones semiestructuradas y no estructuradas.

\section{REFERENCIAS BIBLIOGRÁFICAS}

Sprague y Carlson Building Effetive Decision Support System. Englewood Cliffs, NJ, PrenticeHall Inc, 1982.

Peter G.W. Keen Michael S. Scott Morton (1978) "Decision Support System" Addison - Wwsley Pub. Co., California.

Jhiro Amaya Amaya (1992) “Toma de Decisiones Gerenciales" Ecue Ediciones, Colombia. 